\title{
REFLEXIONES SOBRE EL VALOR DOCENTE DE UNA COMPETICION DE DRONES EN LA EDUCACIÓN PARA EL CONTROL
}

\author{
Ignacio Díaz Blanco, Álvaro Escanciano Urigüen, Antonio Robles Álvarez, Hilario López García \\ Área de Ingeniería de Sistemas y Automática, Universidad de Oviedo \\ Campus universitario de Gijón s/n, 33204 \\ \{idiaz, arobles, hilario\}@uniovi.es, escanciano.alvaro@gmail.com
}

\section{Resumen}

En este artículo se plantea una reflexión sobre el potencial valor docente en el ámbito de la ingeniería y particularmente en la ingeniería de control de las competiciones de drones y se describen las características de una competición de drones presentada en las XXXVIII Jornadas de Automática (2017). La participación en una carrera de drones como la presentada en esta edición, requiere por parte de los participantes un despliegue de competencias de carácter multidisciplinar, que involucran conocimientos de control, electrónica, mecánica y aerodinámica, entre otros, tanto para el montaje del dron, como para optimizar su comportamiento de cara a la prueba. Un planteamiento adecuado y la integración eficiente de estos elementos puede aportar una considerable ventaja en la prueba. A lo largo del texto se hace una reflexión sobre todos estos aspectos, mostrando las ventajas en el ámbito de la educación en el control que puede aportar este tipo de pruebas.

Palabras clave: Educación, ingeniería de control, drones

\section{INTRODUCCIÓN}

Hace apenas medio siglo, el mundo del aeromodelismo estaba reservado para unos pocos privilegiados que, combinando fuertes conocimientos teóricos con una gran capacidad práctica a la hora de construir prototipos, conseguían a duras penas unos minutos de vuelo. Con el paso del tiempo y la llegada de la era digital, la electrónica cada vez se fue reduciendo, no solo en tamaño sino también en coste y esto permitió introducir una gran cantidad de componentes electrónicos en un espacio muy reducido, con poco peso y al alcance de todos los bolsillos. En en mundo del aeromodelismo, la irrupción de esta electrónica (pequeña y barata), supuso la posibilidad de sustituir la estabilización mecánica que proporciona el efecto giroscópico de la máquina de vuelo rotativo más famosa (el helicóptero) por una estabilización electrónica, mucho más compleja y basada en el control independiente de cada uno de los 4, 6, 8 ó 12 motores de un multirotor, que se denomina comúnmente dron.

La posibilidad de construir a bajo coste un dispositivo extremadamente versátil como el dron, y en todas sus etapas, conlleva una innumerable cantidad de decisiones de diseño que implican la puesta en práctica de conocimientos de un amplio espectro de disciplinas con especial protagonismo de la ingeniería de control, la integración de múltiples sistemas en una unidad eficiente y la aplicación de una metodología que implica diseño, montaje y pruebas de verificación. Todos estos ingredientes, que están en el ADN de la ingeniería de sistemas y automática, conforman un escenario óptimo en la formación de ingenieros de nuestra rama de conocimiento.

En el marco de las presentes XXXVIII Jornadas de Automática, se planteó llevar a cabo un concurso de drones, en aras de contribuir al objetivo de conseguir un mayor conocimiento e implicación de alumnos de último curso de grado y máster en temas de ingeniería de control, aunque también en otros aspectos de ingeniería, como la electrónica, el diseño aerodinámico, etc. La competición se realiza con cuadricópteros y hexacópteros con características de partida similares, incluyendo pruebas de habilidad, velocidad y máxima distancia en vuelo recorrida con una sola carga de batería.

De forma análoga a las competiciones de Fórmula 1, una competición de drones en diversas modalidades, como la que aquí se plantea, conlleva una importante componente tecnológica de carácter multidisciplinar, que implica la optimización de los drones desde cualquier aspecto que el participante considere ventajoso: aerodinámica, ajuste de los sistemas de control de vuelo (PID), equilibrado mecánico, etc. donde una adecuada optimización del dron, aportaría ventajas en varios niveles: menor consumo, mayores prestaciones dinámicas, estabilidad y maniobrabilidad, entre otras.

\section{ANTECEDENTES}

\subsection{La ludificación en la educación}

La idea sobre la que se reflexiona en este trabajo, en la que se plantea el análisis de una competi- 
ción de drones en cuanto a su valor docente en el ámbito de la ingeniería de control y de sistemas, tiene conexiones con el concepto de ludificación (del inglés, gamification). El término ludificación describe el uso de elementos de juego en contextos de no-juego, con el objetivo de involucrar a la gente en una variedad de tareas [5]. Uno de los elementos clave en la ludificación es la competición. La competición implica alcanzar un objetivo en un contexto de contienda entre varios rivales (individuos o equipos) donde generalmente se da un perdedor y un ganador. Aunque los beneficios en el ámbito de la educación del elemento de competición han sido objeto de cierta controversia [2] (por ejemplo, focalización en el objetivo y no en el proceso, o el estrés y desmotivación en caso de malos resultados) muchos estudios se inclinan por los beneficios, vinculándola con las ideas de motivación, reconocimiento, desafío y el fomento del aprendizaje activo.

La idea que se analiza aquí, como se verá más adelante, implica una competición en 4 modalidades (carrera, eficiencia, velocidad y diseño), por lo que las expectativas de fracaso son menores, al haber más opciones para el éxito. Por otra parte, la participación es por equipos, por lo que el impacto de una derrota es potencialmente menor al ser compartido.

\subsection{Experiencias anteriores: carreras de drones}

Un claro antecedente de la idea que se analiza en este artículo son las carreras de drones. En este sentido, la participación en el campeonato de España, celebrado en octubre de 2016 en Barcelona, sirve como referencia. En esta prueba pudo comprobarse ya el impacto de la ingeniería en la consecución de una ventaja competitiva en la consecución del éxito. La conclusión de los organizadores fue que no existe una configuración perfecta para un dron, sino más bien una configuración óptima para una prueba concreta en unas condiciones determinadas y en un momento de la competición conocido. Cada nueva parte del "hardware" del dron que se sustituye, impacta en la configuración de la aeronave y el control debe ser nuevamente reconfigurado.

Esta experiencia y otras carreras celebradas a nivel local ponen de manifiesto que el diseño, ajuste y parametrización del dron, aspectos estrechamente ligados a los paradigmas de la ingeniería de sistemas y dela ingeniería de control, tienen un impacto significativo en los resultados obtenidos en la prueba que, cuando menos, rivaliza con el factor de habilidad en el pilotaje.

\section{VALOR EDUCATIVO EN INGENIERÍA DE CONTROL}

\subsection{METODOLOGÍA}

\subsubsection{Flujo de trabajo}

El diseño y construcción de un dron, especialmente si se destina a una competición, requiere al estudiante poner en práctica una metodología de optimización y diseño, basada en elaboración de hipótesis, prueba y error. Dependiendo del grado de dedicación, podrá atacar el diseño a varios niveles: diseño preliminar de controladores; simulación, empleando herramientas como simulink u otros más específicos como gacebo; pruebas reales con prototipo; y despliegue final (carrera).

Aunque existen textos excelentes sobre metodología de diseño de sistemas de control (ver, [3], cap. 10), la mera puesta en práctica de este flujo de trabajo le hará llegar, por sus propios medios, a muchas de las recomendaciones de diseño descritas en la literatura y le resultarán obvias al final del proceso. En definitiva, le transmiten, por inmersión, varios conceptos difícilmente transmisibles por medio de un estudio meramente teórico a través de los textos. Así, por ejemplo, podrá comprobar la existencia de múltiples grados de libertad en el diseño, la necesidad de encontrar un balance óptimo entre los mismos y la importancia del ciclo planificación-test. Todo ello tiene un enorme valor educativo de carácter transversal en la ingeniería de sistemas y control, que constituye una excelente formación complementaria a la impartida en los estudios reglados.

\subsubsection{Codiseño de proceso y control}

Otro aspecto de importancia en control, fuertemente ligado a la metodología, es el diseño integrado (codiseño) del proceso y el control $[1,4]$. En el diseño de sistemas de control, a menudo las modificaciones en la planta son una alternativa ventajosa frente las modificaciones en el propio sistema de control. Esta cuestión se ha planteado en sistemas aerodinámicos, en los que puede mejorarse la maniobrabilidad haciendo el diseño aerodinámico deliberadamente inestable [6] y delegando en el control para recuperar la estabilidad del conjunto [1]. Este enfoque está en la base del desempeño y maniobrabilidad que muestran los aviones de combate modernos.

En el diseño de drones a pequeña escala enfocados a carreras, o a competiciones específicas como la que se presenta en estas Jornadas, esta posibilidad se ve catalizada por la implantación y accesibilidad hoy en día de tecnologías de impresión 3D y las herramientas de simulación de elemen- 
tos finitos, que brindan infinitas posibilidades de optimización en el diseño:

- Reducción del peso en el chasis, eliminando secciones del mismo que contribuyen poco a la resistencia mecánica del dron a partir de los resultados del cálculo de esfuerzos bajo simulación.

- Modificaciones en el chasis y otros elementos optimizando comportamiento aerodinámico, que faciliten el control dinámico de estabilidad del dron.

- Selección óptima de la posición y orientación de los elementos de propulsión (actuadores). En este sentido, por ejemplo, ya se han descrito configuraciones orientadas a carreras y competiciones, situando las hélices en dos niveles y con orientaciones específicas [7].

\subsubsection{Grados de libertad en el diseño}

Otra idea que se percibe en el diseño de un dron es que el control aporta grados de libertad en la ingeniería. El control permite modular el comportamiento de un sistema tecnológico dado. Además de aportar estabilidad (como en el caso de un segway), permite diseñar a medida la maniobrabilidad de un sistema. Aún sin conocimientos de teoría de control, los pilotos de drones son conscientes de que los conceptos de estabilidad y maniobrabilidad están, por propia naturaleza, reñidos. Si lo que se busca es un vuelo de precisión, tranquilo, estable, la ejecución de una ruta programada vía GPS o la filmación de un video de gran calidad, el modo de vuelo adecuado a configurar sería el "modo estable", modo en el que toda la sensorización se pone al servicio del control y se dota al aparato de una gran estabilidad (sacrificando tiempos de respuesta y maniobrabilidad). Por el contrario, cuando lo que se desea es configurar una aeronave competitiva capaz de dejar a todas las demás atrás en una carrera mediante la ejecución de giros cerrados, loopings, tirabuzones y descensos en picado el modo correcto de vuelo a configurar, sería el denominado "modo acrobático", en el que se desactiva toda la sensorización del dron y el control únicamente actúa como interfaz entre el piloto y la aeronave pero en ningún caso estabiliza la misma, es el piloto el que debe hacerlo. En este modo de vuelo, se prioriza, por tanto, la maniobrabilidad por encima de la estabilidad.

\subsection{SISTEMAS DE CONTROL}

Además de la metodología, que implica beneficios educativos de carácter transversal -aunque fuertemente ligados al control-, el diseño de un dron tiene una componente de alto valor específico en ingeniería de control que es el propio diseño y ajuste de los sistemas de control.

Los sistemas de control están en el centro del diseño del dron y permiten mantener estable el dron a partir de la información aportada por los sensores (giróscopos o acelerómetros). Los frameworks típicos para programación del dron, constan habitualmente de un firmware, que se carga en la controladora del dron y que contiene la planificacion de tareas y los lazos de estabilización y control del dron, y un software que permite la carga o transferencia del firmware, así como la modificación de parámetros y reglajes del mismo desde un ordenador. El software permite el ajuste de una amplísima variedad de parámetros, incluyendo acciones P, I, D para varios lazos independientes, como el ángulo o velocidad angular en los ejes roll, pitch y yaw (alabeo, cabeceo y guiñada), pero también multitud de ajustes adicionales, como la sensibilidad de los mandos o sistemas como el TPA (throttle PID attenuation), que reduce la intensidad de las acciones de control para valores elevados en la referencia, para evitar oscilaciones. La variedad de ajustes es extensísima y diferente en función del software/firmware empleado.

A la hora de diseñar el control de un dron, el usuario puede optar por el caso de menor complejidad, cargando en la controladora del dron los modos de vuelo por defecto, pudiendo reajustar a partir de ellos de forma independiente las acciones de control y resto de parámetros para los distintos lazos.

No obstante, en el caso de que los modos de vuelo preconfigurados no sean suficientes, existen infinidad de opciones de modificación del diseño. Los paquetes software de controladores de vuelo más extendidos son de código abierto, destacando algunos como cleanflight [8], o librepilot [9], ambos soportando gran variedad de controladoras y configuraciones de drones.

Al tratarse de herramientas de código abierto (por ejemplo, cleanflight está en github y librepilot puede encontrarse en bitbucket), es posible entrar a nivel de código para editar el existente o incluso crear nuevos programas y bucles de control que serán compilados y cargados a la controladora a través del software proporcionado por el fabricante. Esta última opción permitiría abordar virtualmente cualquier paradigma de control del estado del arte (control multivariable, esquemas adaptativos, control predictivo, etc.). Así, podrían, por ejemplo, plantearse esquemas híbridos, con distintos "modos de control" entre los que el dron podría conmutar en pleno vuelo, bien comandadas por el piloto, o bien de forma automática, en función de las condiciones de vuelo o de la prueba. 


\subsection{ASPECTOS TECNOLÓGICOS}

El diseño del dron, finalmente, tiene una importantísima faceta tecnológica. El control está intrínsecamente ligado a los bloques físicos: sensores, actuadores y planta. La intensa componente tecnológica y de ingeniería que implica el diseño y el montaje un dron comporta dificultades y situaciones específicas que difícilmente pueden describirse en textos teóricos. En esta sección describimos, a modo de ejemplo, algunos de ellos:

\subsubsection{Montaje del dron}

Supone un procedimiento común en ingeniería: consultar información técnica, implementar un sistema y probarlo. En el montaje del dron cabe incluir también el diseño del frame (chasis) mediante impresión 3D, mecanizado del carbono, etc.

\subsubsection{Características y dimensiones del dron}

La elección y dimensionado del frame tiene también varias implicaciones que requieren una elección óptima. El tamaño incrementa el peso, pero admite hélices mayores. Por otra parte el material del que esté hecho el frame, influirá también en el peso y además en la resistencia mecánica, que confiere ventajas en carrera en caso de impacto. Estudio del efecto de la geometría, distancia entre ejes, etc.

\subsubsection{Hélices}

Selección de las hélices (bipala, tripala). La elección del tipo y dimensiones de las hélices determina el empuje tanto absoluto como específico en función de las revoluciones. El tamaño de las hélices, asimismo, condiciona el tamaño del frame y por tanto el peso, por lo que implica alcanzar un balance óptimo. Finalmente, el número de palas tiene una influencia en características como la velocidad punta, aceleración y maniobrabilidad (siendo las bipala mejores en las dos primeras y las tripala superiores en la tercera).

\subsubsection{Circuitos estabilizadores de corriente}

Efectos en el rendimiento del dron. Posible impacto en el consumo de batería.

\section{CARACTERÍSTICAS DE LA PRUEBA}

En la sección anterior se han hecho reflexiones y expuesto algunas de las múltiples facetas de valor docente que comporta el diseño y montaje de un dron, por ejemplo para una competición convencional. Sin embargo, las competiciones de drones, en general, no están planteadas con un objetivo docente y, más concretamente, en el ámbito de la ingeniería de control. En este artículo se plantea una reflexión sobre la posibilidad de introducir algunas modificaciones y elementos específicos a una competición convencional con el objeto de incrementar su potencial valor educativo en el ámbito del control. Con este objeto, se describen algunas ideas generales, basadas en la experiencia de diseño de una competición de drones para las XXXVIII Jornadas de Automática con ingredientes específicos para alcanzar dicho propósito, con el objeto de que sirvan de utilidad o de guía para otras experiencias.

\subsection{TIPOS DE DRON}

Para asegurar la homogeneidad de la prueba, es conveniente establecer limitaciones al tipo y dimensiones de los drones y/o de sus componentes, incluyendo limitaciones de tamaño (medidas de frames), peso total, tipo de baterías, etc.

\subsection{PRUEBAS}

\subsubsection{Carrera en un circuito}

La prueba de carrera es, probablemente, la más exigente, requiriendo habilidad en el pilotaje, velocidad de respuesta dinámica, estabilidad y maniobrabilidad. La carrera de drones puede planificarse con dinámica similar a la de una competición convencional, pero con algunos ingredientes específicos (obstáculos, túneles con geometrías difíciles, viento, etc.) que permitirán evaluar la maniobrabilidad y estabilidad en vuelo de los drones, conseguidas con el "tuneado" del dron (electrónica, aerodinámica, geometría) y el ajuste de los sistemas de control del dron.

\subsubsection{Máxima distancia recorrida en vuelo}

Con este tipo de prueba se plantea evaluar la eficiencia en el consumo obtenida gracias al diseño y el ajuste de los sistemas de control, frente a la "eficacia" en carrera. La prueba puede diseñarse computando la máxima distancia que es capaz de recorrer en vuelo un dron con una sola batería cargada, en un circuito pequeño y sencillo que no suponga un reto para que, de esta forma, se prime la faceta de eficiencia.

\subsubsection{Velocidad entre dos puntos}

Esta modalidad persigue poner a prueba la respuesta dinámica en el dron, el control de potencia 
y la precisión para aterrizar en un punto. La prueba puede basarse en la realización un "Sprint" en línea recta, con despegue desde suelo, contabilizando el tiempo empleado en llegar desde la línea de salida a la línea de meta, aterrizando en un área dada.

Esta prueba, que supone una intensa demanda de corriente en la batería, podría combinarse con la prueba de eficiencia anterior, lo que plantea mayor dificultad en la optimización del conjunto.

\subsubsection{METODOLOGÍA Y DISEÑO}

Este apartado tiene como objetivo evaluar la metodología aplicada y las decisiones en el diseño. Para ello, cada participante o equipo deberá elaborar un documento de diseño, en el que deberá describir todo el proceso (elección de los componentes, el diseño de los controladores, aspectos relativos a la integración de los sistemas, etc.) y argumentar el porqué de las decisiones adoptadas.

Los documentos pueden ser sometidos a un proceso de revisión por un comité, que valore de forma global la calidad de los documentos (argumentos, decisiones de diseño, presentación formal, legibilidad, etc.). Asimismo, el comité podrá recabar in situ, durante las fases de prueba, información acerca de los diseños y comprobar de primera mano cualquier aspecto que permita complementar las valoraciones. Finalmente, el comité los ordenará en un ranking.

\subsection{SISTEMA DE PARTICIPACIÓN CRUZADA}

Uno de los retos de la prueba es el de aislar la componente de habilidad de la componente técnica en el resultado de la prueba. Los resultados de la competición, o al menos una parte de ellos, deben poder reflejar el rendimiento, maniobrabilidad y respuesta dinámica del dron de forma independiente de la habilidad del piloto. Una posibilidad es un sistema de participación cruzada, en el que cada dron sea pilotado por un número $n$ de participantes, elegidos por sorteo, incluyendo el participante que presenta el dron. De esta manera, es posible evaluar de forma independiente el rendimiento de ambos agregando los resultados obtenidos en las pruebas por piloto y por dron.

\subsection{RANKING}

A partir de las pruebas obtenidas, puede establecerse un ranking global de acuerdo con el siguiente procedimiento:

- Se calculará la puntuación total de cada equipo sumando el valor ordinal de los puestos al- canzados en cada una de las cuatro categorías $(4.2 .1,4.2 .2,4.2 .3,4.2 .4)$. El primer puesto suma 1 punto, el segundo puesto suma 2 puntos y así sucesivamente.

Cuando un equipo no finalice una prueba se le asignará en dicha categoría una puntuación igual al número total $n$ de equipos inscritos.

- Las puntuaciones totales obtenidas por cada equipo determinarán su posición en el ranking de la prueba, siendo el ganador absoluto de la prueba aquél que haya obtenido menor puntuación y asignándose los puestos sucesivos por orden creciente de puntos.

- En caso de empate a puntos entre dos o más equipos, su orden en el ranking se determinará de acuerdo con el puesto obtenido en la categoría de metodología y diseño (sección 4.2.4).

Este ranking, similar al que hay en otras pruebas deportivas (ej. la "combinada" en la vuelta ciclista), supone una función de coste multiobjetivo que premia a los equipos "más completos", es decir, aquellos con resultados buenos en todas las pruebas.

\subsection{PREMIOS Y DIPLOMAS}

Los premios y el reconocimiento a los participantes con mejores resultados juegan un papel esencial en el proceso, ya que introduce objetivos que aportan motivación. La posibilidad de otorgar premios o distinciones en distintas categorías permite que los participantes tengan más opciones de éxito, mejorando sus expectativas y motivación. En la competición celebrada en las Jornadas se otorgan premios y diplomas en las categorías: absoluta (mejor ranking absoluto), metodología, carrera, eficiencia, velocidad.

\section{Evaluación de resultados}

\subsection{Encuesta a los participantes}

Una vía para evaluar el impacto de la prueba es la realización de encuestas a los propios participantes, basándose en la hipótesis de que el estudiante, especialmente en últimos cursos, tiene ya una cierta capacidad para percibir que ha aprendido algo y ha incrementado sus conocimientos. Aunque el grado de autopercepción de lo aprendido pueda ser una medida poco fiable en lo cuantitativo, sí es más confiable a un nivel binario ("aprendî" / "no aprendî") o con un número bajo de niveles ("poco", "algo", "mucho"). Bajo esta hipótesis, la 
agregación estadística de resultados puede aportar una medida cuantitativa más confiable, especialmente si el tamaño muestral es significativo.

La forma más adecuada de valorar tanto el grado de satisfacción de los alumnos con el evento como los conocimientos adquiridos durante el transcurso del mismo será la creación de encuestas con campos a determinar por los docentes organizadores de las pruebas. Estas encuestas serán complementadas también con informes del proceso iterativo que ha seguido cada equipo hasta configurar su aeronave para las distintas pruebas.

\subsection{Reportes de diseño}

Los reportes del proceso iterativo que ha seguido cada equipo hasta configurar cada aeronave para cada prueba también pueden aportar información sobre el valor docente de la la prueba. No obstante, a diferencia de la encuesta, extraer indicios objetivos y cuantificables de los reportes es mucho más difícil. No se dispone propiamente de un elemento de referencia o comparación que permita valorar un incremento en el aprendizaje, salvo, quizás informes anteriores de los mismos alumnos realizados en competiciones o actividades similares. Los reportes, sin embargo, sí aportan información útil en otros aspectos. Por ejemplo, puede tenerse una percepción a nivel absoluto (no relativo) del nivel de los conocimientos y capacidades desplegadas para la prueba, que podrían aportar conclusiones sobre los puntos fuertes que fomenta la competición. Por otra parte, lo visto en los reportes puede jugar un importante papel de complementariedad, en combinación con las encuestas, que aporte un refuerzo y validación en las conclusiones extraídas de éstas.

Finalmente, si hay disponibilidad, la comparación de estos reportes con exámenes o trabajos previos de los alumnos puede enriquecer la comparación y valoración de lo aportado por la participación en la prueba.

\section{CONCLUSIONES Y FUTURO TRABAJO}

En este trabajo se ha pretendido dar una visión de las posibilidades del uso de competiciones o pruebas similares con drones desde dos aspectos: 1) el diseño, montaje, prueba y despliegue de un dron como actividad altamente educativa en aspectos multidisciplinares de ingeniería y especialmente en ingeniería de sistemas y control; y 2) el valor educativo de la competición en sí misma, como estímulo y como herramienta de validación del rendimiento que puede verse, en un sentido amplio, como una "función de coste", así como algunas pautas y sugerencias para "modularla" a través de las bases de la competición, introduciendo especificidades para maximizar el peso de la componente técnica.

Finalmente, debemos indicar que las ideas expuestas en este trabajo son reflexiones elaboradas durante la preparación de la prueba y basadas en la información y experiencia adquirida en el proceso. Los resultados de la competición de drones no están disponibles en el momento de redactar este trabajo. Como futuras líneas de trabajo se plantean la elaboración de encuestas de participación y su posterior análisis y valoración.

\section{Referencias}

[1] K. J. Aström. Control System Design. Lecture notes for ME 155A. Department of Mechanical and Environmental Engineering. University of California Santa Barbara., 2003.

[2] I. Cantador and J. M. Conde. Effects of competition in education: A case study in an elearning environment. In Proceedings of the IADIS International Conference E-learning, 2010.

[3] G. F. Franklin, J. D. Powell, and A. EmamiNaeini. Feedback Control of Dynamic Systems. Pearson Prentice Hall, 5th edition edition, 2006.

[4] K. J. Aström and R. M. Murray. Feedback Systems: An Introduction for Scientists and Engineers. Princeton University Press, March 2010.

[5] S. de Sousa Borges, V. H. Durelli, H. M. Reis, and S. Isotani. A systematic mapping on gamification applied to education. In Proceedings of the 29th Annual ACM Symposium on Applied Computing, pages 216-222. ACM, 2014.

[6] G. Stein. Respect the unstable. IEEE Control Systems Magazine, 23(4):12-25, Aug. 2003.

[7] William Thielicke Aerodynamics in racing multirotors. Why future racing copters really should look different. https://tinyurl.com/y7scmhfq Sep. 26,2015

[8] http://cleanflight.com

[9] https://www.librepilot.org/site/index.html 